\title{
Characterization of PAGAT dose response upon different irradiation
}

conditions

G. Magugliani*, G. M. Liosi, D. Tagliabue, E. Mossini, M. Negrin, M. Mariani

Department of Energy, Politecnico di Milano, Piazza Leonardo da Vinci 32, I - 20133 Milano, Italy

*e-mail: gabriele.magugliani@ @ail.polimi.it 


\title{
Characterization of PAGAT dose response upon different irradiation conditions
}

\author{
Polymer gel dosimeters represent a promising instrument for tridimensional \\ radiation dose measurement for highly conformal radiation therapy techniques. In \\ this study, the dependence of the dose response of the polymeric gel dosimeter \\ PAGAT was investigated as a function of different irradiation conditions and \\ time elapsed after irradiation. An X-ray tube and ${ }^{60} \mathrm{Co}$ sources were used for \\ irradiation. This allowed to assess variations in system dose response under \\ different irradiation energies and dose rates - ranging from $1.4 \mathrm{~Gy} \mathrm{~h}^{-1}$ up to 0.14 \\ $\mathrm{kGy} \mathrm{h}^{-1}$. Analysis of irradiated samples was performed by UV-Vis optical \\ measurements at different time intervals after irradiation. Significant variability \\ in PAGAT dose response was observed for different irradiation conditions and \\ considered dose rates.
}

Keywords: polymer gel dosimeter, PAGAT, dose rate, UV-vis optical analysis

\section{Introduction}

Modern radiotherapy techniques such as Intensity Modulated Radiation Therapy and Stereotactic Radiosurgery allow the delivery of highly conformal radiation dose distributions. This is achieved by combining very steep dose gradients (1) with very precise relative machine-patient positioning. These techniques therefore allow to better preserve radiosensitive organs and healthy tissues surrounding target volumes from excessive and unwanted radiation exposure.

Such high precision in dose delivery, consequently, calls for very accurate, precise and reliable dosimetric instruments for treatment verification. 
To date, commercially available dosimetric systems typically acquire 1D or 2D data through ionization chambers, arrays of semiconductor detectors or photographic films $(2,3)$. Therefore, using these instruments a tridimensional dose map can only be computationally reconstructed. A direct acquisition of tridimensional experimental data would be desirable for a better verification of the calculated treatment plan.

Chemical gel dosimeters represent a valid instrument for clinical 3D dose evaluations in highly conformal radiotherapy treatments $(4,5)$. They present the unique and desirable capability of being arbitrarily shaped and the possibility of simulating tissue inhomogeneity, such as in the case of the pulmonary region (6).

The dose response of chemical dosimeters is based on radiation-induced chemical modifications. Gel-based systems, in particular, allow a 3D mapping of the absorbed dose thanks to the presence of the tissue equivalent gel matrix $(5,7)$.

Among gel systems, polymeric gel dosimeters present several advantages, in particular in terms of high spatial preservation of the acquired dose maps (5) and limited dependence of the dose response on irradiation energy (7). Their dose response originates from the radiation induced free radical polymerization of monomers dispersed into a tissue equivalent gel matrix. Upon irradiation, monomers polymerize into cross-linked nanometric aggregates (5), which tend to grow in size proportionally with absorbed dose.

Early dosimeter formulations were sensitive to the strong radical scavenging properties of oxygen, thus requiring preparation in an inert environment (8). In recent years, the so called normoxic compositions have been introduced $(9,10)$, allowing for preparation under normal atmospheric conditions (11).

These systems are capable of 3D retention of delivered dose, since polymeric 
aggregates present a very limited diffusion inside the gelatin matrix due to their molecular size.

However, despite the low mobility of polymer chains, a loss of spatial information on absorbed dose is nevertheless observed (12). The main contribution to the temporal degradation of spatial resolution originates from the slow diffusion of unreacted monomers that can progress polymerization even after irradiation has terminated. These are so called diffusion-controlled termination reactions $(13,14)$, which in general can have very long timescales (days-months), thus presenting some challenges for prolonged dosimeter storage.

Magnetic Resonance Imaging (MRI) has great potentiality for 3D imaging of polymer gel dosimeters, thanks to its intrinsic tridimensional probing capability. Physical response of polymer gel dosimeters originates from a decrease in proton spinspin relaxation time $\mathrm{T}_{2}$ with absorbed dose (13).

MRI allows data acquisition with a sensitivity and spatial resolution $(15,16)$ adequate for its application in clinical dosimetry; nevertheless, it presents significant disadvantages, mainly due to the limited availability of scanner machine time, which tends to be prioritized towards patient diagnostic.

Optical computed tomography (OCT) was proposed (17) as a cheaper and more flexible alternative to MRI for 3D evaluation of irradiated dosimeters. In particular, it overcomes the limitations deriving from the availability of scanner machine time and costs linked to the acquisition and operation of an MRI apparatus. Its principle of operation is based on the acquisition of multiple 2D optical attenuation projections along various directions, from which a 3D map of the optical attenuation coefficient can be reconstructed (18). 
Since radiotherapy treatments can employ various irradiation energies, dose rates and particles for dose delivery, a comprehensive optical characterization of the response of dosimetric systems with respect to these parameters is necessary. This is an essential step to allow the acquisition of reliable 3D dose distributions through OCT measurements.

In this study, the dose rate-dependent response of the tissue equivalent (19) normoxic polyacrylamide gel dosimeter PAGAT was investigated by means of UV-Vis optical measurements. Optical response of polymeric gel dosimeters is consistent with Mie scattering theory (20), since in the visible range polymeric particles resulting from irradiation present a characteristic dimension comparable to incident light wavelength $(21)$.

Despite PAGAT and other polymeric gel dosimeters analyzed with MRI typically show very limited or negligible dependence of their response on irradiation energy and dose rate $(13,16,22,23)$, no information on the optical response of PAGAT with respect to these parameters can be found in literature - to the best of the authors' knowledge.

\section{Materials and Methods}

\section{Preparation}

All reagents used in this study were purchased from Sigma Aldrich, Italy.

The PAGAT dosimeter consists in an aqueous solution of deionized water, $5 \% \mathrm{w} / \mathrm{w}$ gelatin from porcine skin (gel strength 300, Type A, G2500), 3\% w/w Acrylamide (AA) (for electrophoresis, $\geq 99 \%$, A3553), 3\% w/w N,N'-Methylenebisacrylamide (BIS) (99\%, 146072), and $10 \mathrm{mM}$ THPC (80\% solution in water, 404861). The preparation 
procedure followed in this study is reported hereafter; it is largely similar to some described in literature (24), with minor modifications.

Firstly, gelatin is added to deionized water under stirring and allowed to swell for 5 minutes; the solution is then heated and maintained at $45^{\circ} \mathrm{C}$ to achieve complete dissolution, typically requiring 15 minutes. After the solution appears transparent, AA and BIS are added and allowed to fully dissolve. Heating is then turned off and the solution allowed to cool naturally. At $35^{\circ} \mathrm{C}$ THPC is added drop-wise under gentle stirring to achieve complete homogenization.

The PAGAT solution is then quickly poured into spectrophotometric PMMA cuvettes (4,5 ml capacity, $10 \mathrm{~mm}$ optical path) which are capped, sealed with Parafilm and stored in a refrigerator at $4{ }^{\circ} \mathrm{C}$ for at least 12 hours before irradiation. This time interval is needed to ensure complete and uniform gelification of the solutions. Three separate batches of PAGAT were manufactured for each irradiation setup in order to evaluate the reproducibility of the preparations and of the dose response data.

\section{Irradiation}

Irradiations were performed with the techniques reported below; all dose rate values are to be intended at the effective sample position during irradiation.

- X-ray tube: Seifert model ISOVOLT $320 / 10$ operated at $300 \mathrm{kV}-10 \mathrm{~mA}$, providing a dose rate of $1.4 \mathrm{~Gy} \mathrm{~h}^{-1}$ with $199 \mathrm{keV}$ average energy, $1^{\text {st }} \mathrm{HVL} 5.21$ mm Cu (Calibration facility LAT $n^{\circ} 104$, compliant to EN ISO/IEC 17025:2005, Politecnico di Milano); 
- ${ }^{60} \mathrm{Co}$ irradiator providing dose rates of 0.14 or $0.014 \mathrm{kGy} \mathrm{h}^{-1}$ at the time of the experiments, hereafter referred to as "Low" and "High" dose rate, respectively (Laboratory of Applied Nuclear Energy, University of Pavia).

The X-ray tube is equipped with on-line irradiation control performed with a transmission ionization chamber with aluminized Mylar windows, coupled with a galvanometer for absolute charge measurement.

For the ${ }^{60} \mathrm{Co}$ irradiations, the dosimeters were placed in the region of greatest field uniformity. The effective delivered dose rates were previously measured by means of thermoluminescent dosimeters (TLDs) embedded into non-dosimetric gel samples providing the same geometry and irradiation configuration as in the case of the PAGAT dosimeters. These measurements allowed to establish a field uniformity greater than $95 \%$ in the irradiation region.

${ }^{60} \mathrm{Co}$ was selected over more conventional MV LINAC irradiation since its continuous dose delivery allows a more direct comparison with X-Ray response without introducing additional variables in irradiation parameters. Moreover, as already discussed, polymer gels do not show significant dependence on irradiation energy, so the choice of ${ }^{60} \mathrm{Co}$ energy should not limit the generality of the obtained results.

All samples were irradiated in free air; the following doses for each irradiation method were delivered:

- X-rays: from 1 to 3 Gy with a step increase of $1 \mathrm{~Gy}$;

- Low dose rate ${ }^{60}$ Co source: from 1 to 6 Gy with a step increase of $1 \mathrm{~Gy}$;

- High dose rate ${ }^{60}$ Co source: from 2 to $10 \mathrm{~Gy}$ with a step increase of $2 \mathrm{~Gy}$. 
Since the planned time for X-ray irradiation was quite long (more than 2 hours to deliver $3 \mathrm{~Gy}$ ), a maximum absorbed dose of $3 \mathrm{~Gy}$ was selected, in order to limit the superposition of the radiation induced polymerization with the underlying diffusiondriven polymerization.

In the case of the High dose rate ${ }^{60} \mathrm{Co}$ source irradiation, as verified by means of the TLD calibration mentioned above, the minimum step dose increase which guarantees a sufficient accuracy and precision (lower than 1\%) in dose delivery is $2 \mathrm{~Gy}$. Hence the dose repartition in the High dose rate ${ }^{60}$ Co irradiation.

For each irradiation setup, three samples from three different preparation batches were irradiated at the same dose to quantify the reproducibility of the acquired data.

\section{Optical measurement and data analysis}

Optical absorbance measurements were performed with a LAMBDA $650 \mathrm{UV} / \mathrm{V}$ is spectrophotometer (PerkinElmer, Italy), employing a non-irradiated sample from each batch as blank reference. Following a previous optimization of acquisition parameters, $550 \mathrm{~nm}$ was identified as the optimum wavelength for absorbance evaluation. Prior to measurements samples were allowed to reach room temperature $\left(20 \pm 1{ }^{\circ} \mathrm{C}\right)(5)$. To assess the dose response stability, optical analyses were performed $1 \mathrm{~h}$ and $24 \mathrm{~h}$ post irradiation.

The sensitivity of each system is defined as the slope of the absorbance vs dose linear relation; dose resolution was calculated, for a 95\% level of confidence, as (25):

$$
\text { Dose resolution }=\frac{2.77 \bar{\sigma}}{S}
$$

Where $\mathrm{S}$ is the sensitivity and $\bar{\sigma}$ is the arithmetic mean of standard deviation on dose. 
All figures reported in this work show mean sample values with symmetric error bars representing one standard deviation of uncertainty.

\section{Results}

Linearity of response was confirmed for each irradiation setup up to the highest delivered dose of $6 \mathrm{~Gy}$; above this dose, in the case of High dose rate ${ }^{60} \mathrm{Co}$ irradiation, a saturation in response is noticeable (Figure 3).

Only in the case of irradiation with the Low dose rate ${ }^{60}$ Co source a dose response threshold of 2 Gy was observed (Figure 4), after which a linear response up to 6 Gy followed.

[Insert Figure 4 near here]

In the case of X-ray irradiation, a non-ideal linearity was observed $1 \mathrm{~h}$ post irradiation, which improves over 24 h to yield a good linear fit $\left(R^{2}>0,99\right.$, Figure 1$)$.

A significant increase in sensitivity inversely proportional to dose rate was noticed, amounting to $75 \%$ for X-rays when compared to the High dose rate ${ }^{60} \mathrm{Co}$ irradiation.

Values of sensitivity and dose resolution for the PAGAT dosimeter analyzed at 1 and 24 $\mathrm{h}$ post-irradiation are summarized in Table 1 and graphically represented in Figures from 1 to 3 .

[Insert Table 1 near here] 
[Insert Figures 1, 2, 3 near here]

\section{Discussion}

Optical analysis performed at $24 \mathrm{~h}$ post irradiation provided better results in terms of sensitivity and dose resolution; this was expected, since it is well known that polymer gel dosimeters require time to achieve complete development of the signal (10). For this reason, the comparisons and discussion hereafter were conducted among parameters measured at $24 \mathrm{~h}$ post irradiation.

As previously mentioned, PAGAT presents an almost constant energy absorption coefficient for energies employed in this work (7), so it is assumed that irradiation energy plays a minor role on measured response variability.

Dose resolution values showed no major dependence on irradiation parameters. Their variation can be attributed mainly to intrinsic reproducibility among different dosimeter batches.

A response saturation could only be noticed in the case of the High dose rate ${ }^{60} \mathrm{Co}$ irradiation above $6 \mathrm{~Gy}$, since such high doses were not investigated in the case of the lower dose rates due to constraints in irradiation length as described earlier. It is nevertheless expected that a saturation would be present at doses higher than the ones delivered.

The increase in sensitivity observed in the case of Low dose rate X-ray irradiation (Figure 5) may be attributed to two concurring effects: firstly, the prolonged exposure times, on the order of hours in this study, and secondly the dose rate. 
[Insert Figure 5 near here]

It has been observed that pre-irradiated polymer dosimeters show an increase in response to a further irradiation session (26); it is theorized that this behavior is a consequence of the abundance of reactive sites in the polymeric network that can further react with monomers in solution. In the case of a prolonged irradiation a similar behavior is expected. In fact, in the latter stages of irradiation, a polymeric network has already developed inside the gel, thus providing a positive enhancement on the response by the same mechanism that would be present in case of a pre-irradiation.

This matrix effect would become less important with the increase of dose rate and shortening of irradiation times, as in the case of the two ${ }^{60} \mathrm{Co}$ irradiations.

Secondly, a lower irradiation dose rate may cause a lower instantaneous concentration of radical monomers, and therefore a less probable termination by recombination in the early stages of propagation, leading to the formation of larger polymeric aggregates. This hypothesis would be coherent with the results obtained in this study; in fact, according to Mie scattering theory, a larger mean particle size implies a greater scattering cross section, i.e. an increase in measured optical absorbance (27).

The slower achievement of a fully linear response for X-ray irradiation is a sign of incomplete polymerization at the first measurement point at $1 \mathrm{~h}$ post irradiation. This behavior may be coherent with the hypothesis of larger polymeric particles formation. These would present a lower surface to volume ratio, and therefore reactive sites embedded deeply inside these regions would react more slowly due to the local increase in viscosity which would limit diffusion of fresh monomers. On the other hand, smaller 
particles tend to exhaust reactive sites more quickly, therefore reaching stabilization more rapidly, as is seen in particular for the High dose rate ${ }^{60} \mathrm{Co}$ irradiation.

Regarding the dose threshold encountered in the Low dose rate ${ }^{60}$ Co irradiation, acquired data is yet insufficient to identify a specific cause to this behavior. To the best of the authors' knowledge, no studies of PAGAT optical response over the dose rates investigated in this study are available in literature for comparison. It may be possible that such inhibition at low doses was compensated by the enhancement in response in the case of X-ray irradiation, thus not being visible in the latter case.

In the case of High dose rate ${ }^{60} \mathrm{Co}$ irradiation, the minimum delivered dose of $2 \mathrm{~Gy}$ coincides with the threshold observed for the Low dose rate source. Therefore, in this case it is not possible to exclude a response inhibition at doses lower than $2 \mathrm{~Gy}$.

\section{Conclusion}

This study provided novel insights into the optical behavior of a PAGAT dosimeter to varying irradiation conditions. A linear optical dose response in the range of investigated doses was confirmed for each irradiation method, allowing the definition of the characterizing dosimetric quantities of sensitivity and dose resolution. Significant variations in sensitivity with irradiation dose rate were encountered. This irradiation parameter seems to influence especially the optical response of the dosimeter, since MRI evaluations from literature did not find such behavior.

This response variability should be accounted for in case of prolonged irradiations, as it may be the case for dose evaluations in Low Dose Rate brachytherapy, where dose rates below $2 \mathrm{~Gy} \mathrm{~h}^{-1}$ are employed. 
It is theorized that such response variability may be a consequence of prolonged irradiation times and higher radical concentration due to high dose rates. The relative importance of these two effects towards final increase in sensitivity is still unclear.

Further analyses employing more combinations of irradiation energies and dose rates will allow to acquire a more comprehensive understanding of the response of these systems; performing irradiation with a LINAC would allow to assess possible influences of pulsed dose delivery towards optical response. Moreover, a measurement of polymer particle size distribution may help in quantifying the effect of dose rate towards this quantity.

Further investigations will be undertaken to better understand the effect of the polymeric matrix on the dose response. More in detail, subsequent irradiations with 1 Gy steps doses with a high dose rate source will be performed. Delivering subsequent irradiations with such small step dose increase interspersed with adequate resting periods up to the same doses delivered by X-rays, may allow to confirm whether the polymer matrix is in fact responsible for the positive effect towards dose sensitivity. This dose delivery repartition would create a polymeric network during each irradiation step, which, given an appropriate time to develop, could interact with its chemical surroundings during the subsequent step dose deliveries. If the interpretation given in this work is correct, i.e. that the enhancement in response is due to the preexisting polymeric matrix, this should yield the same effect as if the irradiation were carried out in a single session but with a lower dose rate source.

\section{Acknowledgements}

The authors would like to acknowledge professor Caresana (Politecnico di Milano, 
Department of Energy) and professor Dondi (University of Pavia, Department of Chemistry) for their contribution in sample irradiations.

\section{References}

(1) Paddick, I.; Lippitz, B. A simple dose gradient measurement tool to complement the conformity index. J. Neurosurg. 2006, 105, 194-201.

(2) Sadagopan, R.; Bencomo, J. A.; Martin, R. L.; Nilsson, G.; Matzen, T.; Balter, P. A. Characterization and Clinical Evaluation of a Novel IMRT Quality Assurance System. J. Appl. Clin. Med. Phys. 2009, 10 (2), 104-119.

(3) Ramani, R.; Lightstone, A. W.; Mason, D. L. D.; O’Brien, P. F. The Use of Radiochromic Film in Treatment Verification of Dynamic Stereotactic Radiosurgery. Med. Phys. 1994, 21 (3), 389-392.

(4) Oldham, M.; Siewerdsen, J. H.; Shetty, A.; Jaffray, D. A. High Resolution GelDosimetry by Optical-CT and MR Scanning. Med. Phys. 2001, 28 (7), 1436-1445.

(5) Baldock, C.; De Deene, Y.; Doran, S.; Ibbott, G.; Jirasek, A.; Lepage, M.; McAuley, K. B.; Oldham, M.; Schreiner, L. J. Polymer Gel Dosimetry. Phys. Med. Biol. 2010, 55 (5), R1-R63.

(6) Deene, Y. D.; Vandecasteele, J.; Vercauteren, T. Low-Density Polymer Gel Dosimeters for 3D Radiation Dosimetry in the Thoracic Region: A Preliminary Study. J. Phys.: Conf. Ser. 2013, 444, 12026.

(7) Schreiner, L. J. Review of Fricke Gel Dosimeters. J. Phys.: Conf. Ser. 2004, 3, 921. 
(8) Maryanski, M. J.; Schulz, R. J.; Ibbott, G. S.; Gatenby, J. C.; Xie, J.; Horton, D.;

Gore, J. C. Magnetic Resonance Imaging of Radiation Dose Distributions Using a Polymer-Gel Dosimeter. Phys. Med. Biol. 1994, 39 (9), 1437-1455.

(9) Deene, Y. D.; Hurley, C.; Venning, A.; Vergote, K.; Mather, M.; Healy, B. J.;

Baldock, C. A Basic Study of Some Normoxic Polymer Gel Dosimeters. Phys. Med. Biol. 2002, 47 (19), 3441-3463.

(10) Senden, R. J.; Jean, P. D.; McAuley, K. B.; Schreiner, L. J. Polymer Gel

Dosimeters with Reduced Toxicity: A Preliminary Investigation of the NMR and Optical Dose-response Using Different Monomers. Phys. Med. Biol. 2006, 51 (14), $3301-3314$.

(11) Fong, P. M.; Keil, D. C.; Does, M. D.; Gore, J. C. Polymer Gels for Magnetic Resonance Imaging of Radiation Dose Distributions at Normal Room Atmosphere. Phys. Med. Biol. 2001, 46 (12), 3105-3113.

(12) Deene, Y. D.; Venning, A.; Hurley, C.; Healy, B. J.; Baldock, C. Dose-response Stability and Integrity of the Dose Distribution of Various Polymer Gel Dosimeters. Phys. Med. Biol. 2002, 47 (14), 2459-2470.

(13) Deene, Y. D. Essential Characteristics of Polymer Gel Dosimeters. J. Phys.: Conf. Ser. 2004, 3, 34-57.

(14) McAuley, K. B. The Chemistry and Physics of Polyacrylamide Gel Dosimeters: Why They Do and Don’t Work. J. Phys.: Conf. Ser. 2004, 3, $29-33$.

(15) Deene, Y. D. Review of Quantitative MRI Principles for Gel Dosimetry. J. Phys.: Conf. Ser. 2009, 164, 12033. 
(16) Lepage, M.; Whittaker, A. K.; Rintoul, L.; Bäck, S. Å. J.; Baldock, C. The Relationship between Radiation-Induced Chemical Processes and Transverse Relaxation Times in Polymer Gel Dosimeters. Phys. Med. Biol. 2001, 46 (4), 10611074.

(17) Gore, J. C.; Ranade, M.; Maryañski, M. J.; Schulz, R. J. Radiation Dose Distributions in Three Dimensions from Tomographic Optical Density Scanning of Polymer Gels: I. Development of an Optical Scanner. Phys. Med. Biol. 1996, 41 (12), 2695-2704.

(18) Doran, S. J. The History and Principles of Optical Computed Tomography for Scanning 3-D Radiation Dosimeters: 2008 Update. J. Phys.: Conf. Ser. 2009, 164, 12020.

(19) Taylor, M. L.; Franich, R. D.; Trapp, J. V.; Johnston, P. N. The Effective Atomic Number of Dosimetric Gels. Australas. Phys. Eng. Sci. Med. 2008, 31 (2), 131-138.

(20) Maryañski, M. J.; Zastavker, Y. Z.; Gore, J. C. Radiation Dose Distributions in Three Dimensions from Tomographic Optical Density Scanning of Polymer Gels: II. Optical Properties of the BANG Polymer Gel. Phys. Med. Biol. 1996, 41 (12), 27052717.

(21) Oldham, M.; Siewerdsen, J. H.; Kumar, S.; Wong, J.; Jaffray, D. A. Optical-CT Gel-Dosimetry I: Basic Investigations. Med. Phys. 2003, 30 (4), 623-634.

(22) Deene, Y. D.; Vergote, K.; Claeys, C.; Wagter, C. D. The Fundamental Radiation Properties of Normoxic Polymer Gel Dosimeters: A Comparison between a Methacrylic Acid Based Gel and Acrylamide Based Gels. Phys. Med. Biol. 2006, 51 (3), 653-673. 
(23) Zehtabian, M.; Faghihi, R.; Zahmatkesh, M. H.; Meigooni, A. S.; Mosleh-Shirazi, M. A.; Mehdizadeh, S.; Sina, S.; Bagheri, S. Investigation of the Dose Rate Dependency of the PAGAT Gel Dosimeter at Low Dose Rates. Radiat. Meas. 2012, 47 (2), 139-144.

(24) Venning, A. J.; Hill, B.; Brindha, S.; Healy, B. J.; Baldock, C. Investigation of the PAGAT Polymer Gel Dosimeter Using Magnetic Resonance Imaging. Phys. Med. Biol. 2005, 50 (16), 3875-3888.

(25) Deene, Y. D.; Baldock, C. Optimization of Multiple Spin-echo Sequences for 3D Polymer Gel Dosimetry. Phys. Med. Biol. 2002, 47 (17), 3117-3141.

(26) Jirasek, A. I.; Duzenli, C. Effects of Crosslinker Fraction in Polymer Gel Dosimeters Using FT Raman Spectroscopy. Phys. Med. Biol. 2001, 46 (7), 1949-1961.

(27) Graaff, R.; Aarnoudse, J. G.; Zijp, J. R.; Sloot, P. M. A.; de Mul, F. F. M.; Greve, J.; Koelink, M. H. Reduced Light-Scattering Properties for Mixtures of Spherical Particles: A Simple Approximation Derived from Mie Calculations. Appl. Opt. 1992, 31 (10), 1370. 
Table 1. Summary of sensitivity and dose resolution values for PAGAT for each irradiation setup; measurements were performed $1 \mathrm{~h}$ and $24 \mathrm{~h}$ post irradiation.

Confidence intervals represent one standard deviation of uncertainty among three sample batches.

\begin{tabular}{|l|l|l|l|l|}
\hline PAGAT & Post irr. time & X-ray & ${ }^{60}$ Co, Low & ${ }^{60}$ Co, High \\
& & & dose rate & dose rate \\
\hline Sensitivity & $1 \mathrm{~h}$ & $0.191 \pm 0.026$ & $0.150 \pm 0.004$ & $0.187 \pm 0.011$ \\
\cline { 2 - 5 }$\left[\mathrm{Gy}^{-1}\right]$ & $24 \mathrm{~h}$ & $0.327 \pm 0.016$ & $0.182 \pm 0.048$ & $0.156 \pm 0.011$ \\
\hline Dose res & $1 \mathrm{~h}$ & $0.196 \pm 0.041$ & $0.321 \pm 0.081$ & $0.184 \pm 0.047$ \\
\cline { 2 - 5 }$[\mathrm{Gy}]$ & $24 \mathrm{~h}$ & $0.154 \pm 0.049$ & $0.295 \pm 0.044$ & $0.167 \pm 0.047$ \\
\hline
\end{tabular}




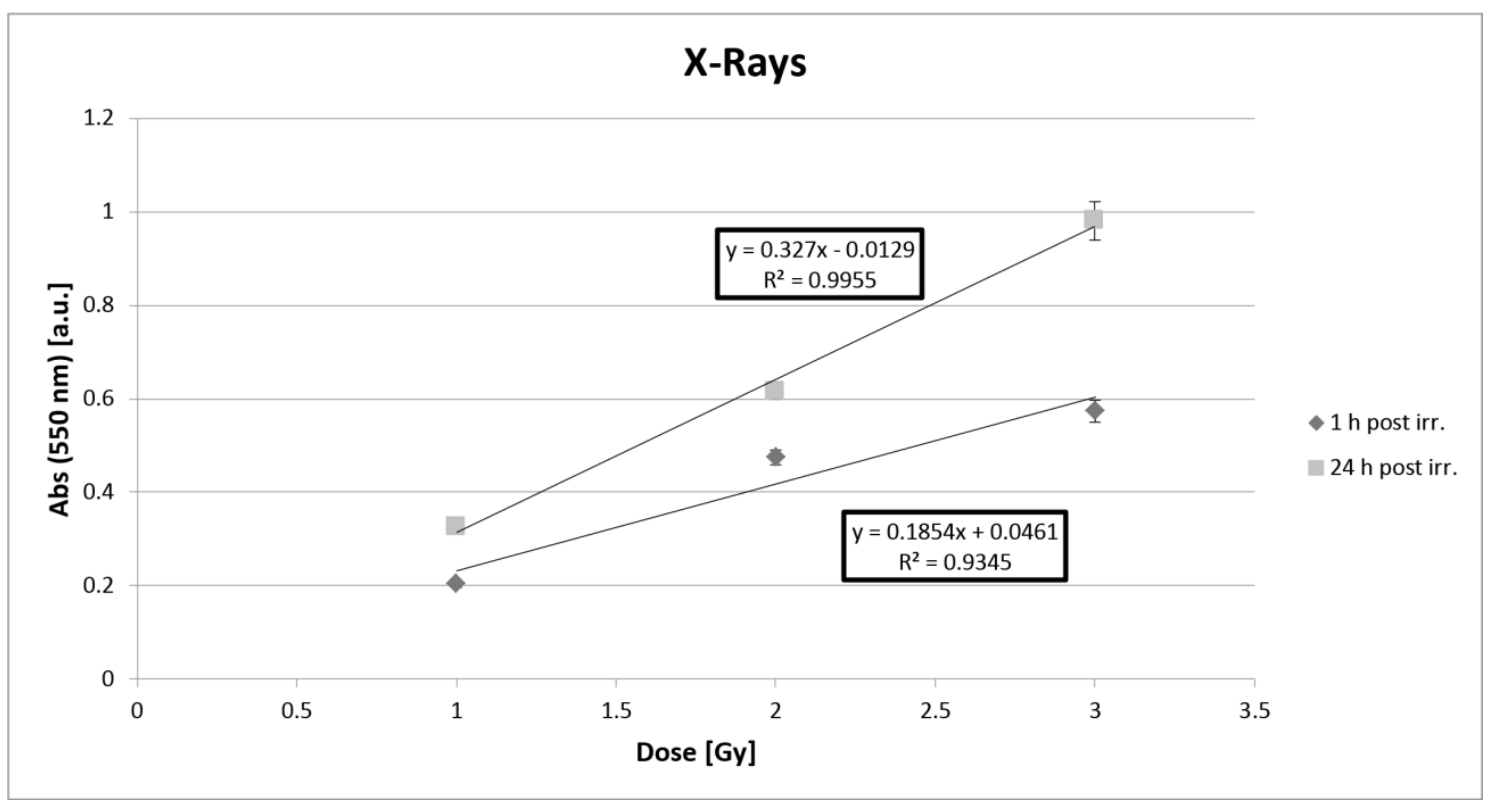

Figure 1: Optical dose response of PAGAT after irradiation with X-ray source. Analyses performed $1 \mathrm{~h}$ and $24 \mathrm{~h}$ post irradiation. 


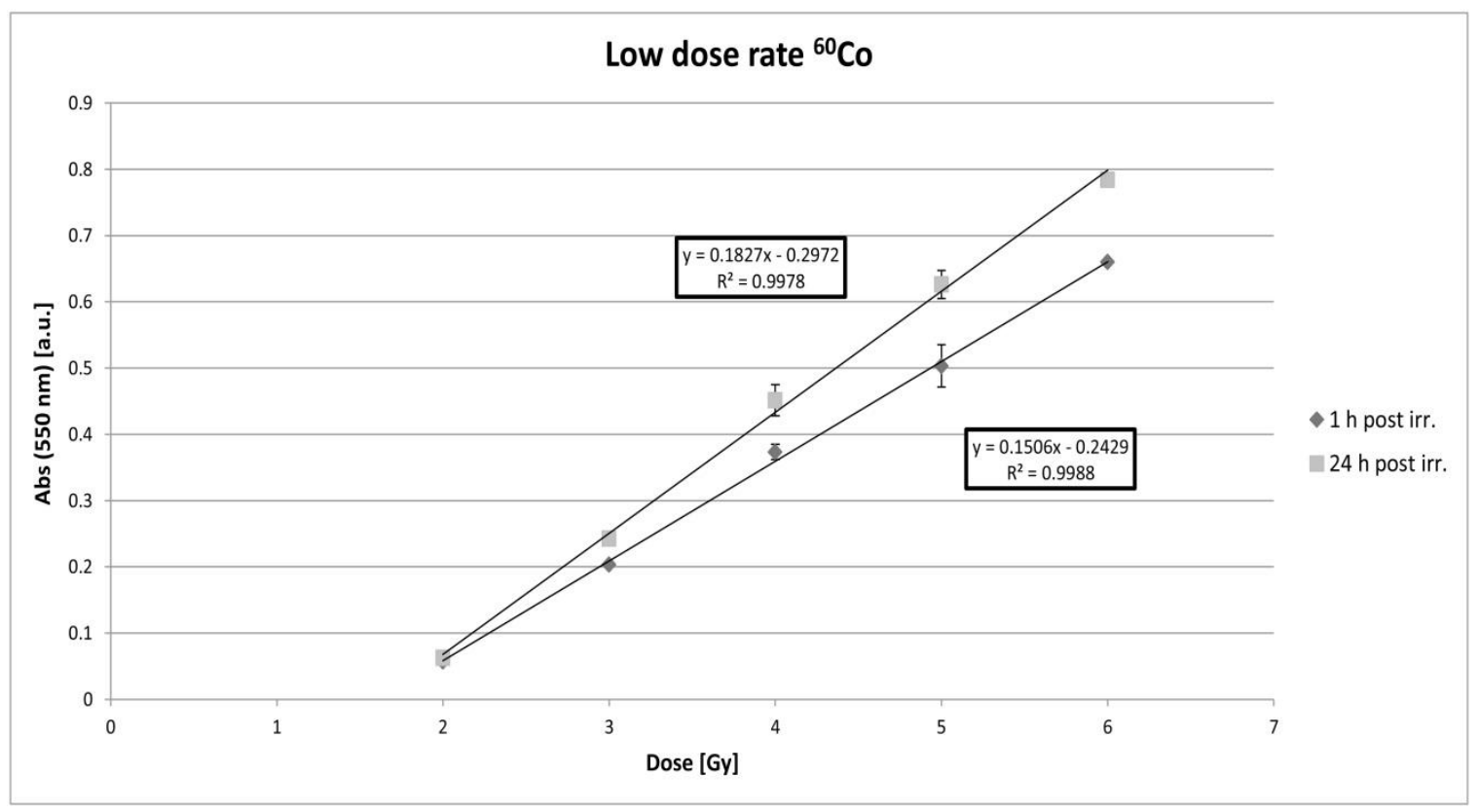

Figure 2: Optical dose response of PAGAT after irradiation with Low dose rate ${ }^{60} \mathrm{Co}$ source. Only the range of linear response is reported. Analyses were performed $1 \mathrm{~h}$ and $24 \mathrm{~h}$ post irradiation. 


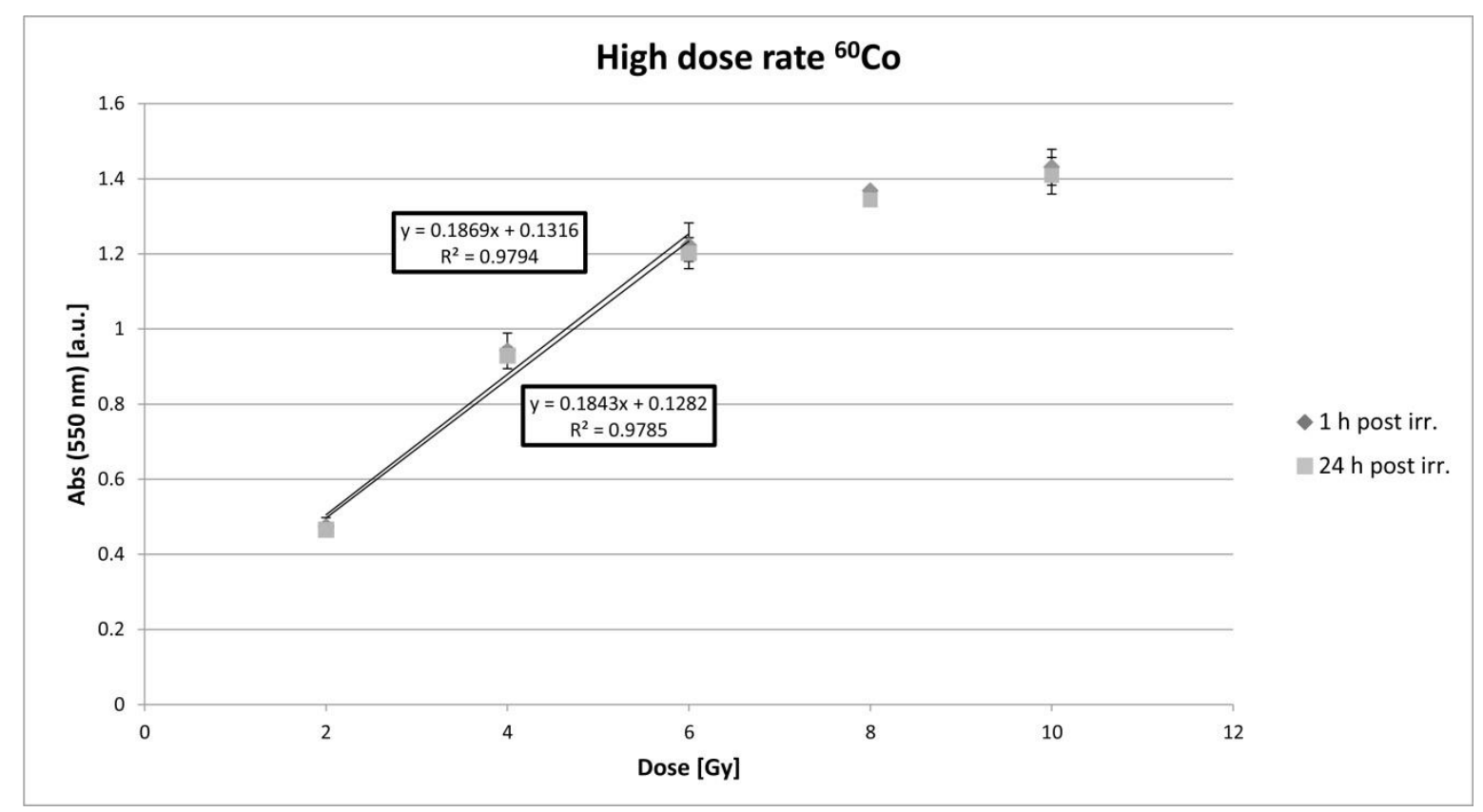

Figure 3: Optical dose response of PAGAT after irradiation with High dose rate ${ }^{60} \mathrm{Co}$ source. Response saturation is noticeable above $6 \mathrm{~Gy}$. Analyses were performed $1 \mathrm{~h}$ and $24 \mathrm{~h}$ post irradiation. 


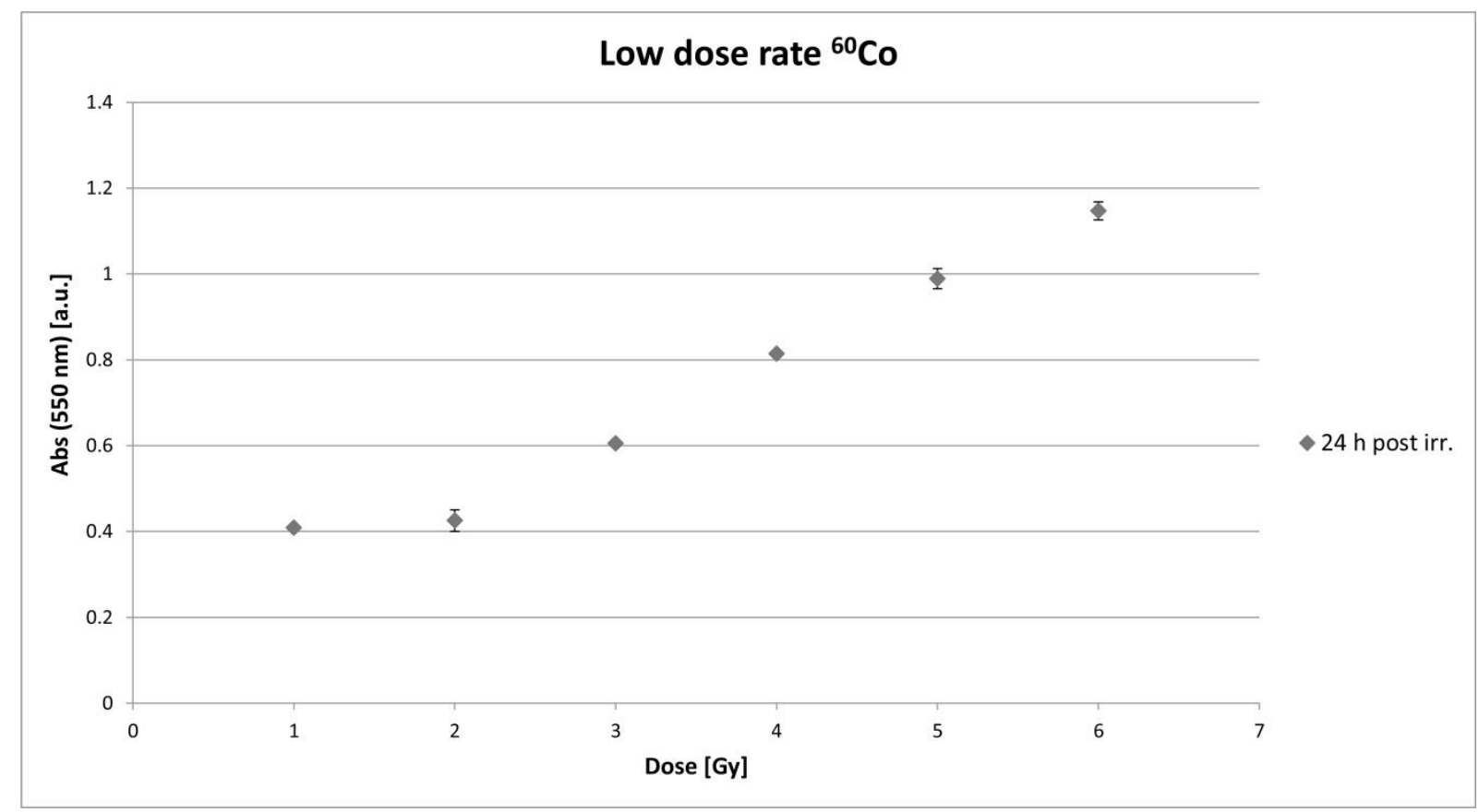

Figure 4: Optical dose response of PAGAT after irradiation with Low dose rate ${ }^{60} \mathrm{Co}$ source, analyzed $24 \mathrm{~h}$ post irradiation. A dose response threshold is present for doses up to $2 \mathrm{~Gy}$. 


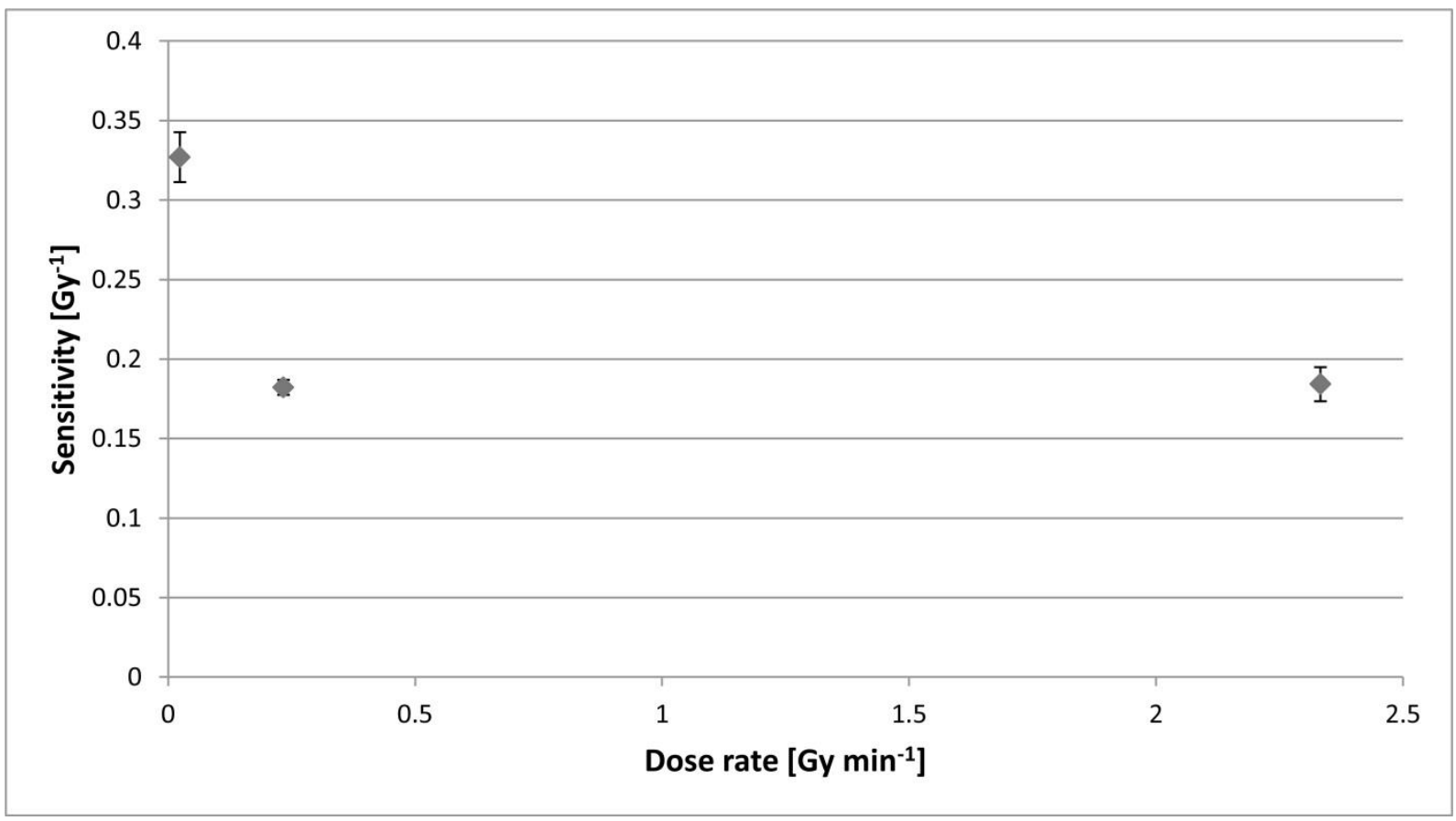

Figure 5: PAGAT dose sensitivity variation with irradiation dose rate. 